\section{THU0380 DIAGNOSTIC UTILITY OF ANTI-DFS-70 AUTOANTIBODIES IN A UNIVERSITY RHEUMATOLOGY CENTRE}

U. Salzer, A.C. Venhoff, I. Jandova, R.E. Voll, J. Thiel, N. Venhoff. Department of Rheumatology and Clinical Immunology, University Medical Center Freiburg, Freiburg, Germany

Background: The detection of antinuclear autoantibodies by immunofluorescence (ANA-IFT) supports the diagnosis of many different autoimmune and rheumatological diseases (ARE). In combination with the detection of specific autoantibodies against known extracted nuclear antigens (ENA) ANAs have high diagnostic sensitivity and specificity. However, positive ANA-IFT may also occur in disease states not related to ARE and even in healthy individuals. Especially in the latter group this may lead to unnecessary and repeated hospital visits, as well as avoidable diagnostic and therapeutic interventions. Anti-DFS70 autoantibodies were introduced as a biomarker for the exclusion of ARE in ANA-IFT + patients without additional ENAs and non-specific clinical history.

Objectives: To evaluate if the diagnostic pathway for suspected or established ARE patients in our centre benefits from the addition of anti-DFS70 autoantibodies to an existing ENA test profile.

Methods: Serum from patients was tested for anti-DFS70 autoantibodies by the ANA-Profil 3 plus DFS70 line blot (Euroimmun, Lübeck Germany).

Results: In 2017 the Freiburg university rheumatology centre tested 671 patients, referred to for diagnosis or follow up of ARE, for anti-DFS70 and 126 patients were found positive. Descriptive statistics of the anti-DFS70 positive vs negative cohorts are summarised in table 1 . Of the 126 anti-DFS70 positive patients, 53 $(42 \%)$ had one or more additional ENAs positively tested (4 snRNP/Sm, $1 \mathrm{Sm}, 15$ SS-A/Ro, 15 Ro-52,2 SS-B/La,4 Scl-70,8 PM-Scl,2 PM-Scl75,1 SRP,2 Ku, 1 Jo1,4 Centromer,2 PCNA,2 Nukleosomes, 9 Histones,1 ribos-P-Prot,2 AMA-M2,6 ds-DNA) and $3(2 \%)$ patients had anti-DFS70 in conjunction with anti CCP autoantibodies. $70(56 \%)$ patients were tested single positive for anti-DFS70. 81 (64\%) of anti-DFS70 positive patients either had an established diagnosis of ARE or were newly diagnosed with ARE based on clinical criteria and laboratory results. The majority of these patients had additional ENAs or other autoantibodies tested positive, as described above. A diagnosis of ARE was excluded or revised with help of the anti-DFS70 result in $45(36 \%)$ patients, all of these were anti-DFS70 positive only. In 10 of these patients (20\%) preexisting therapies were discontinued (9 Hydroxychloroquin, 1 MTX/sulfasalazine).

\begin{tabular}{lccc}
\hline & $\begin{array}{c}\text { Anti-DFS70 positive } \\
(\mathbf{n}=\mathbf{1 2 6})\end{array}$ & $\begin{array}{c}\text { Anti-DFS70 negative } \\
(\mathbf{n}=\mathbf{5 4 4 )}\end{array}$ & p-value \\
\hline Male, $\mathrm{n}(\%)$ & $19(15.1)$ & $111(20.4)$ & n.s. \\
Mean age, years (min-max) & $47(18-77)$ & $52(14-88)$ & n.s. \\
DFS70 +++, n (\%) & $82(65.1)$ & NA & NA \\
DFS70 ++, n (\%) & $13(10.3)$ & NA & NA \\
DFS70 +, n (\%) & $10(7.9)$ & NA & NA \\
DFS70 $(+), \mathrm{n}(\%)$ & $21(16.7)$ & NA & NA \\
\hline
\end{tabular}

Abstract THU0380 - Table 1. Descriptive statistics of the patient cohorts tested for antiDFS70-antibodies

Conclusions: We found a high added diagnostical value of anti-DFS70 autoantibodies testing, since they were helpful to rule out or revise a previously suspected diagnosis of ARE in more than a third all patients tested anti-DFS70 positive at our centre. However, patients referred to a university rheumatology centre are a highly selected cohort and have a higher probability being finally diagnosed with ARE, and thus consideration of clinical criteria and thorough testing for additional autoantibodies is recommended in such a setting since also many patients with confirmed ARE will test positive for anti-DFS70.

Disclosure of Interest: None declared

DOI: 10.1136/annrheumdis-2018-eular.6479

\section{THU0381 HIGH LEVELS OF CIRCULATING TYPE I, II AND III INTERFERONS DEFINE DISTINCT PATIENT SUBSETS WITH ACTIVE SYSTEMIC LUPUS ERYTHEMATOSUS (SLE)}

${ }^{1}$ V. Oke, I. Gunnarsson ${ }^{1}$, J.M. Dorschner ${ }^{2}$, A. Zickert ${ }^{1}$, T.B. Niewold ${ }^{3}$, E. Svenungsson ${ }^{1} .{ }^{1}$ Rheumatology Unit, Department of Medicine, Karolinska Institutet, Stockholm, Sweden; ${ }^{2}$ Division of Rheumatology and Department of Immunology, Mayo Clinic, Rochester, $\mathrm{MN} ;{ }^{3}$ Colton Center for Autoimmunity, New York University, New York, NY, USA

Background: Interferons (IFN) play a major role in SLE pathogenesis.' IFNs type I (predominately IFN- $\alpha$ ) are of major importance, ${ }^{2}$ but IFN type II (IFN- $\gamma$ ) and IFNs type III $(\lambda)$ have also important roles. ${ }^{3,4}$ How the levels of circulating IFNs type I, type II and type III relate to each other, and if they associate with any particular SLE disease manifestations is not known.
Objectives: We investigated serum levels of type I, type II, and type III IFNs and explored how these measurements relate to each other and to specific organ manifestations in patients with SLE.

Methods: We studied 497 well-characterised SLE patients and 322 controls. Functional type I IFN-activity (IFN-activity) was measured by WISH cell assay. IFN- $\alpha$ and IFN- $\lambda 1$ were measured by ELISA, and IFN- $\gamma$ by MSD 30 -plex assay. High IFN-activity/levels were defined as value over 3rd quartile of the measurement.

Results: SLE patients had higher levels of all investigated IFNs. IFN-activity correlated with IFN- $\alpha$ and IFN- $\gamma$. High functional IFN-activity associated with active SLE in most domains: weight loss, fatigue, fever, rash, lymphadenopathy, arthritis and nephritis. The IFN- $\gamma$ high group had active disease with higher rates of nephritis, arthritis, leuko-, lymphopenia and Sm, SmRNP, RNP68, Ro52 and Ro60 autoantibodies. A higher proportion of the IFN- $\alpha$ high group had active rash lymphadenopathy, Ro52 and La autoantibodies, while rates of antiphospholipid antibodies/syndrome, vascular events and renal affection were lower. High IFN- $\lambda$ 1 associated with anti-nucleosome autoantibodies and lymphopenia.

Conclusions: High type I IFN functional activity is associated with active SLE in the majority domains. A severe SLE phenotype, including active nephritis, arthritis and anti-Sm/SmRNP autoantibodies is associated with high IFN- $\gamma$, while rash and a benign cardiovascular profile are linked to high serum IFN- $\alpha$. Isolated increase in IFN- $\lambda 1$ is only coupled to lymphopenia and antinucleosome antibodies. Our findings demonstrate that several IFNs can be elevated at the same time in SLE and the importance of IFN- $\gamma$ has so far been underscored. Sub-setting of SLE patients might be important when planning future clinical trials.

\section{REFERENCES:}

[1] Niewold TB, Hua J, Lehman TJA, et al. High serum IFN-alpha activity is a heritable risk factor for systemic lupus erythematosus. Genes Immun 2007;8:492-502. doi:10.1038/sj.gene.6364408

[2] Bengtsson AA, Sturfelt G, Truedsson L, et al. Activation of type I interferon system in systemic lupus erythematosus correlates with disease activity but not with antiretroviral antibodies. Lupus 2000;9:664-71.

[3] Oke V, Brauner S, Larsson A, et al. IFN- $\lambda 1$ with Th17 axis cytokines and IFN- $\alpha$ define different subsets in systemic lupus erythematosus (SLE). Arthritis Res Ther 2017:19:139. doi:10.1186/s13075-017-1344-7

[4] Machold KP, Smolen JS. Interferon-gamma induced exacerbation of sys temic lupus erythematosus. J Rheumatol 1990;17:831-2.

Acknowledgements: We are grateful to Susanna Eketjäll, Eva Jemseby, Johanna Gustafsson, Marie Wahren-Herlenius, Susanna Brauner, Ola Börjesson, Marika Kvarnström, Susanne Pettersson, Sonia Möller, Jill Gustafsson for help with different parts of the project.

Disclosure of Interest: None declared

DOI: 10.1136/annrheumdis-2018-eular.4783

\section{THU0382 FATIGUE IN CHINESE PATIENTS WITH SYSTEMIC LUPUS ERYTHEMATOSUS: CONTRIBUTING FACTORS AND EFFECTS ON THE QUALITY OF LIFE}

${ }^{1}$ X. Du, Y. Zhuang ${ }^{1}$, Q. Zhao ${ }^{1}$, H. Chen ${ }^{2}$, B. Shen ${ }^{1} .{ }^{1}$ The Second Affiliated Hospital of Nantong University, Nursing College of Nantong University, ${ }^{2}$ The Second Affiliated Hospital of Nantong University, Nantong, China

Background: Fatigue is a very common symptom in Systemic Lupus Erythematosus (SLE), affecting more than $90 \%$ of patients ${ }^{[1]}$. Fatigue can lead to a decline in the quality of life ${ }^{[2]}$. Fatigue in SLE patients is associated with adverse demographic, clinical, and psychological characteristics ${ }^{[3,4]}$. However, there is no systematic study of SLE fatigue in China.

Objectives: This cross-sectional study aims to evaluate the contributors of fatigue and the effects of fatigue on the quality of life in Chinese SLE patients. Methods: A self-report survey was administered to 119 SLE patients and 105 healthy individuals using the Fatigue Severity Scale(FSS) to assess the severity of fatigue. SLE patients completed the Systemic Lupus Erythematosus Disease Activity Index (SLEDAI) for disease activity, the Hospital Anxiety and Depression Scale(HADS) for anxiety and depression, the Pittsburgh Sleep Quality Index (PSQI) for sleep quality and the Short Form 36 health survey for the quality of life. Meanwhile, healthy individuals completed the Hospital Anxiety and Depression Scale(HADS) and the Pittsburgh Sleep Quality Index (PSQI). We used Independent samples t-tests, Chi square analysis and logistic regression modelling to analyse these data.

Results: Our results found that the FSS score of patients with SLE was higher than that of the controls $(4.33 \pm 1.66$ versus $3.41 \pm 1.39 ; p<0.001)$. The SLE patients were significantly different from the control group in terms of anxiety, depression and quality of life. There were significant correlations among course of disease, anxiety, depression, subjective sleep quality, sleep disorders and fatique in SLE patients. Meanwhile, logistic regression models identified depression and 